\title{
Client and provider factors associated with integration of family planning services among maternal and reproductive health clients in Kigoma Region, Tanzania: a cross- sectional study, April-July 2016
}

\author{
M. M. Dynes ${ }^{1 *}$ (D) E. Bernstein ${ }^{1}$, D. Morof ${ }^{1}$, L. Kelly ${ }^{1}$, A. Ruiz², W. Mongo ${ }^{3}$, P. Chaote ${ }^{4}$, R. N. Bujari ${ }^{5}$ and F. Serbanescu ${ }^{1}$
}

\begin{abstract}
Background: Integration of family planning (FP) services into non-FP care visits is an essential strategy for reducing maternal and neonatal mortality through reduction of short birth intervals and unplanned pregnancies.

Methods: Cross-sectional surveys were conducted across 61 facilities in Kigoma Region, Tanzania, April-July 2016. Multilevel, mixed effects logistic regression analyses were conducted on matched data from providers $(n=330)$ and clients seeking delivery $(n=935)$, well-baby $(n=272)$, pregnancy loss ( $\mathrm{PL} ; n=229)$, and other routine (postnatal, HIV/STI, other; $n=69$ ) services. Outcomes of interest included receipt of FP information and a modern FP method (significance level $p<0.05$ ).

Results: Clients had significantly greater odds of receiving FP information if the primary reason for seeking care was for PL versus (vs) any other types of care (aOR 1.97), had four or more pregnancies vs fewer (aOR 1.78), and had had a FP discussion with their partner vs no FP discussion (aOR 1.73). Clients had lower odds of receiving FP information if they were aged 40-49 vs 15-19 (aOR 0.50) and reported attending religious services at least weekly vs less frequently (aOR 0.61). Clients of providers who perceived that in-service training had helped vs had not helped job performance (aOR 2.27), and clients of providers having high vs low recent FP training index scores (aOR 1.58) had greater odds of receiving FP information.

Clients had greater odds of receiving a modern method when they received information on two or more vs fewer methods (aOR 7.13), had had a FP discussion with their partner vs no discussion (aOR 5.87), if the primary reason for seeking care was for PL vs any other types of care (aOR 4.08), had zero vs one or more live births (aOR 3.92), made their own FP decisions vs not made own FP decisions (aOR 3.17), received FP information from two or more vs fewer sources (aOR 3.12), and were in the middle or high vs the low wealth tercile (aOR 1.99 and 2.30, respectively). Well-baby care clients, Other routine services clients, and married clients had significantly lower odds of receiving a method (aOR 0.14; aOR 0.08; and aOR 0.41, respectively) compared to their counterparts.
\end{abstract}

Conclusions: Strategies that better integrate FP into routine care visits, encourage women to have FP discussions with their partners and providers, increase FP training among providers, and expand FP options and sources of information may help reduce the unmet need for FP, and ultimately lower maternal and neonatal mortality.

Keywords: Family planning, Family planning counseling, Family planning integration, Contraception, Contraceptive methods, Sub-Saharan Africa, Tanzania, Multilevel modeling

\footnotetext{
* Correspondence: mdynes@cdc.gov

${ }^{1}$ Centers for Disease Control and Prevention, Division of Reproductive Health,

Atlanta, USA

Full list of author information is available at the end of the article
}

(c) The Author(s). 2018 Open Access This article is distributed under the terms of the Creative Commons Attribution 4.0 International License (http://creativecommons.org/licenses/by/4.0/), which permits unrestricted use, distribution, and reproduction in any medium, provided you give appropriate credit to the original author(s) and the source, provide a link to the Creative Commons license, and indicate if changes were made. The Creative Commons Public Domain Dedication waiver (http://creativecommons.org/publicdomain/zero/1.0/) applies to the data made available in this article, unless otherwise stated. 


\section{Plain English summary}

Integrating family planning (FP) services into non-FP care visits is an important approach to reduce maternal and newborn deaths through reduction of short birth intervals and unplanned pregnancies. Analyses of matched interviews with 1505 clients and 330 providers in Kigoma Region, Tanzania identified key factors for clients receiving FP information and a modern method. Clients were more likely to receive FP information if they were at the facility for pregnancy loss, had four or more pregnancies, and had discussed FP with their partner. Clients were less likely to receive FP information if they were 40 to 49 years old and attended religious services at least weekly. Providers were more likely to provide FP information if they perceived in-service training had helped their job performance, and had received more recent FP training. Clients were more likely to receive a FP method if they received information on two or more FP methods, had discussed FP with their partner, were at the facility for pregnancy loss, had no live births, made their own FP decisions, received FP information from two or more sources, and were in the middle or high wealth groups. Well-baby care clients, Other services clients, and married clients were less likely to receive a FP method compared to others. FP services need to be better integrated into well-baby and other routine outpatient services. Interventions are needed that encourage women to have FP conversations with their partners and providers, expand FP options and sources of information, and encourage providers to ask about reproductive health goals at each client encounter.

\section{Introduction}

Sub-Saharan Africa experiences disproportionately high maternal mortality in comparison to other regions, contributing to two-thirds of the world's total maternal deaths in 2015 [1]. Tanzania had an estimated 8200 maternal deaths in 2015, the fourth highest number of maternal deaths in sub-Saharan Africa and the sixth highest number in the world [1]. Family planning (FP) can reduce the risk of maternal and neonatal mortality by reducing the number of short birth intervals, as well as unplanned and high-risk pregnancies [2-7]. In 2016, the total fertility rate (TFR) in Tanzania's Western Zone, which includes Kigoma Region, was 6.7 births per woman - the highest TFR in all of Tanzania [8].

Research in Western and African clinical settings has found that quality FP counseling-identified as six distinct elements by the Bruce Framework [9] - has a significant impact on FP knowledge and use [10, 11]. To increase postpartum FP uptake and reduce unmet need, FP counseling and provision are routinely recommended for integration into antenatal, labor and delivery, and postnatal care services [12-17]. However, evidence varies on whether the increase in FP use relates to the type of care services in which FP services are being integrated $[13,23,27]$, number of times counseling is provided [17-20], whether the FP counseling is provided during the antenatal versus postpartum period [15, 17-21], and whether modern contraceptive supplies are available at the time/onsite. Moreover, some studies have found positive effects while others have found no effect [16, 21, 22].

Furthermore, many factors may influence FP uptake even when services are integrated. Necessary infrastructure improvements, increased staff resources, and availability of FP supplies rarely accompany the integration of FP services into other health services $[14,19]$. Long wait times, unclear pathways to FP providers, and lack of privacy deter women from accepting a FP referral [23]. In health facility assessments in Kigoma Region, Tanzania in 2015 , only $57 \%$ of dispensaries had implants and only $39 \%$ had intrauterine devices (IUD) available to clients [24].

\section{Client predictors of FP use}

Women's individual, sociodemographic characteristics, and life circumstances also influence FP uptake. Partner communication and support, self-efficacy, intensity of antenatal care visits, and previous FP use have been found to be positively associated with FP uptake [16, 20, 25]. Lower wealth and history of physical intimate partner violence have been associated with nonuse of FP methods $[12,17,25,26]$. Women's reasons for not using FP include wanting to talk with their partner first [23], waiting until their current baby is older [23], waiting until their menses return [27], and fearing side effects and health risks [28].

\section{Provider predictors of FP use}

Health providers' perceptions and biases may influence provision of services and subsequent likelihood of client FP uptake. Providers may counsel clients against using certain methods or impose medically unfounded barriers to FP methods based on perceived client characteristics including age, partner consent, marital status, and parity [7, 23, 29, 30]. Providers' personal biases have been found to be more commonly imposed for long-term or permanent methods [29], for condoms and pills depending on client age [29] and parity [7], and among providers in private facilities [8] or those with less FP in-service training [7, 29]. In contrast, provider willingness to share personal experiences of FP use [31] and perception of client characteristics, such as education and ability to understand FP options [29], may be positively associated with provider engagement in more directed FP counseling, and may result in greater client satisfaction with services [32].

\section{Reproductive health in Kigoma region Tanzania}

In 2016, Kigoma Region's TFR was 6.5 births per woman, the modern FP prevalence rate among women in union 
was $20 \%$, and the unmet need for FP among women in union was 36\% [33]. There are several national Ministry of Health, Community Development, Gender, Elderly, and Children (MoHCDGEC) ongoing efforts to improve maternal health in Tanzania that have particularly targeted Kigoma Region. These include the National Roadmap Strategic Plan to Accelerate Reduction of Maternal, Newborn and Child Deaths in Tanzania 2008-2015; the Big Results Now (BRN) initiative; and Wazazi Nipendeni ("Parents Love Me"), a mobile health initiative that includes FP and safe motherhood text messages. Since 2006, the Project to Reduce Maternal Deaths in Tanzania has worked in the region with the aim of decreasing maternal mortality through strengthening emergency obstetric and neonatal care and improving access to and use of FP. As part of monitoring and evaluation activities conducted for the Bloomberg Philanthropies-funded Project to Reduce Maternal Deaths in Tanzania, the Centers for Disease Control and Prevention (CDC) has been providing technical assistance to document the integration of FP into health facilities providing maternity care in Kigoma.

\section{Gaps in literature}

Previous studies have explored outcomes of FP counseling and service provision into non-FP services or have described the influence of provider or client characteristics on FP uptake. However, the literature reflects inconsistent associations between receipt of FP information and uptake in methods, and further inconsistencies in the role of client and provider factors in FP integration. Furthermore, there are no published studies on the influence of both client and provider characteristics as predictors of FP integration. This study helps to fill these gaps in the evidence by considering both client and provider characteristics associated with receipt of FP information and modern method among non-FP clients in Kigoma, Tanzania. This information will provide the Tanzanian MoHCDGEC, as well as other stakeholders, with evidence needed to make informed decisions about programmatic and policy implementation for FP integration into maternal and reproductive health services.

\section{Methods}

\section{Study setting and design}

Kigoma Region covers $45,066 \mathrm{~km}^{2}$ and is located in the northwestern Tanzania. In 2012, the region had a population of $2,127,930$; approximately $83 \%$ of the population is designated as rural with farming as the primary economic activity. Nine out of 10 people in Kigoma have attained a primary school education and $76 \%$ of the adult population is literate. [34]

We conducted cross-sectional surveys consisting of facility-based client exit interviews and provider interviews across 61 facilities (6 hospitals, 25 health centers, and 30 dispensaries) in Kigoma Region, Tanzania from April 30 to July 1, 2016.

\section{Sampling and data collection Facility sampling}

All governmental and private hospitals $(n=6)$ and health centers (excluding those in refugee camp settings) $(n=25)$ in Kigoma Region were included. A sample of 30 dispensaries (of the 198 in the region providing delivery services) was selected to maximize geographic distribution and meet the following inclusion criteria: 1) have an estimated 180 or more births per year; 2) have two or more onsite health providers; 3 ) be a site for national or project partner facility improvements, and 4) refer patients to one of the 25 health centers.

\section{Provider and client sampling}

Convenience sampling was used to enroll providers and clients within selected facilities; all providers and clients providing/seeking care on study days were invited to participate if they met inclusion criteria. All types of providers who routinely conducted labor and delivery, pregnancy loss (PL; defined as spontaneous or induced abortion), FP, or postnatal care services were eligible, except for medical doctors and specialists who had limited numbers and availability. Clients were eligible if they were 15 to 49 years of age and received delivery, PL, and routine out-patient (FP, antenatal care [ANC], well-baby, and other) care services at the facility. Clients were excluded if they were younger than 15 or older than 49 years of age, delivered at home or on the way to the facility, had a cesarean section delivery, or experienced a stillbirth or neonatal death.

We determined that 189 provider interviews were sufficient to detect a $5 \%$ relative mean change in key variables of interest related to their knowledge and practice with 90\% power and an alpha of 0.05 . A sample of 908 client interviews was needed to detect a $15 \%$ absolute difference in the variables of interest with $90 \%$ power and an alpha of 0.05 (assuming a 50\% reference proportion). We aimed to interview between two and six delivery and outpatient clients per provider per care type to reduce provider-specific bias; this selection rule was not applied to PL interviews due to the limited availability of PL clients and providers.

\section{Interview procedures}

Questionnaires were pre-tested in January 2016. Final questionnaires were translated from English to Swahili and back-translated to English. Providers were interviewed if they met inclusion criteria and consented to participate. Their clients were approached for participation as they exited care services; clients were asked which care service they received and the corresponding interview guide was administered. All interviews were administered face-to-face by an interviewer in Swahili. 


\section{Study tools}

For client exit interviews, three separate questionnaires were used that were tailored to client service provision type. Questionnaires captured sociodemographic characteristics, perceptions of and satisfaction with services, facility experiences, receipt of FP information and uptake by method, and pregnancy history and intention. Providers completed a questionnaire and knowledge test designed to capture information about provider demographic characteristics, education, training, clinical knowledge, and practices related to labor and delivery care, newborn care, PL, and FP care.

\section{Outcome variables}

In this analysis, we examined two binary outcome variables based on women's self-report: 1) Receipt of FP information (0, did not receive FP information; 1, received FP information); and 2) Receipt of a modern FP method (pill, injection, intrauterine device [IUD], implant, male or female condom, male or female sterilization, or emergency contraception: 0 , did not receive a modern FP method; 1 received a modern FP method).

\section{Independent variables}

Client-level

The client-level variables of interest included:

- Client age (15 to 19, 20 to 29, 30 to 39, 40 to 49 ),

- Delivery care was the primary service sought (yes, no),

- PL care was the primary service sought (yes, no),

- Well-baby care was the primary service sought (yes, no; [immunization, growth]),

- Other routine services was the primary service sought (yes, no; [postnatal care, HIV testing and management, STI]),

- Literacy (able to read and write, able to read or write/neither read nor write),

- Highest education attended (no formal education, primary, secondary, university),

- Number of prior pregnancies (zero to three, four or more),

- Number of live births (zero, one to two, three to five, six or more),

- Marital status (not in union, in union),

- Frequency of attendance at religious services (less than once a week, once a week or more often),

- Wealth (low, middle, high wealth),

- Has heard family planning messages in the last three months (yes, no),

- Desired timing of future childbearing (wants a child in the next two years, wants a child in more than two years/does not want),

- FP discussion with partner (no discussion, has discussed FP with partner),
- FP decision-making (does not make own FP decisions, able to make own FP decisions),

- FP information index (received information about zero or one FP method, received information about two or more FP methods; variable only used for the Receipt of a modern FP method model), and

- FP information source index (received FP information from zero or one source, received FP information from two or more sources; variable only used for the Receipt of a modern FP method model).

The variable Wealth was developed using principal components analysis (PCA) where household assets and characteristics were weighted based on their contribution to the first component and summed to create an index [35]. Each household was given a wealth index score categorized into terciles representing low, middle, and high levels of relative household wealth.

\section{Provider-level}

The provider-level variables of interest included:

- Provider age (younger than the mean of 38 years of age, 38 years or more),

- Sex (male, female),

- Highest education completed (primary, secondary, university),

- Cadre (clinicians [Assistant Medical Officers/Clinical Officers/Assistant Clinical Officers/Clinical Assistants], nurses/midwives [Nurse Officers/ Assistant Nurse Officers/Registered Nurses/ Midwives/Enrolled Nurses], other staff [Medical Attendants/Maternal and Child Health Aides]),

- Years in cadre (less than the mean of 11 years, 11 or more years),

- Years at the facility (less than the mean of eight years, eight years or more),

- Work hours per week (less than the mean of $56 \mathrm{~h}$ per week, 56 or more hours per week),

- Job satisfaction (a little/very satisfied, a little/very dissatisfied/neither satisfied nor dissatisfied),

- Perceptions of fairness in pay (feels he/she is not paid fairly, feels paid fairly),

- Perceptions of adequacy of training (feels training is inadequate for job duties, feels training is adequate),

- Regularly provides FP services (yes, no),

- FP bias (reports no bias against providing FP service, bias reported on one or more client characteristics ${ }^{1}$ ),

- FP recent-training PCA score (low [below mean], high [mean or higher]),

- FP ever-training PCA score (low [below mean], high [mean or higher]),

- FP practice PCA score (low [below mean], high [mean or higher]), 
- Perceptions of helpfulness of in-service training (feels in-service training has not been helpful, feels inservice training has been helpful),

- Electronic mentoring opportunities (no access to elearning/ emergency call system/ teleconference, has access to one or more electronic mentoring activities).

The variables for FP recent-training PCA score (FP training in 2015 or in the first half of 2016), FP ever-training $P C A$ score (FP pre- or in-service training at any time), and FP practice PCA score (current provision of FP services) were developed with PCA using 14 items that cover a range of FP topics. ${ }^{2}$ Elements for recent-training, ever-training, and current practice were weighted based on their contribution to the first principal component and summed to create three separate indices.

\section{Analytic approach}

Client and provider data were linked. Due to the focus of this study on FP integration into non-FP services, FP clients $(n=214)$, current FP users $(n=128)$, and providers who only gave care to FP clients $(n=38)$, were excluded from analyses. ANC $(n=225)$ and other pregnant clients $(n=40)$ were also excluded due to lack of data on desire for future childbearing and FP decision-making.

Data analyses were conducted using Stata 14. Bivariate analyses were conducted to identify client and provider variables associated with the outcome variables of interest. Client and provider variables with an unadjusted association with the outcomes of interest at a level of $p<0.10$ were included in multivariate modeling. A multilevel mixed-effects logistic regression model was used to examine the effects of patient and provider characteristics on either receipt of FP information or receipt of a modern FP method, with random intercepts for each provider. A $p$-value of less than 0.05 was considered statistically significant for multilevel models. We included facility-clustered standard errors to account for unobserved heterogeneity within facilities.

\section{Results}

From April 30-July 1, 2016, 2155 female clients (delivery $n=960$, PL $n=230$, outpatient $n=965$ [inclusive of antenatal, postnatal care, HIV testing and management, STI, and other) and 361 providers were interviewed. Following removal of data from non-linked clients and providers, FP clients, pregnant clients, and current FP users, data from 1505 clients (primary reasons for visit: delivery $n=935$, PL $n=229$, well-baby $n=272$, other routine services $n=69$ ) and 330 providers (clinicians $n=69$, nurses/midwives $n=$ 176 , other staff $n=85$ ) were used in the analysis.

\section{Client and provider characteristics}

Descriptive characteristics of the clients included in the analyses are displayed in Table 1 . The majority of clients were in a union (90.4\%), had attended religious services at least weekly (85.8\%), were able to read and write $(71.1 \%)$, and had attended primary school education (65.8\%). About half of clients were 20 to 29 years of age (49.8\%) and received care at a health center (48.9\%). Two-thirds of clients came to the facility for the purpose of delivery services (62.1\%), while less than one-fifth came for the purpose of well-baby (18.1\%), PL (15.2\%), and other services (4.6\%). Whereas 59.4\% of clients reported having a FP discussion with their partner, less than one-fifth of clients $(17.9 \%)$ reported feeling they are able to make their own FP decisions.

Descriptive characteristics of the providers included in the analyses are displayed in Table 2 . The majority of providers included in the study were female (63.3\%), college/ university educated $(66.7 \%)$, worked in a health center (55.2\%), and were in the nurse/midwife cadre (53.3\%). About two-thirds of providers have worked for more than 10 years in the cadre $(61.5 \%)$ and more than 7 years at their current facility (70\%). Providers worked an average of $54 \mathrm{~h}$ per week, with $57.9 \%$ working more than this average. Less than half of providers were a little or very satisfied with their job (44.9\%), and less than one-fifth of providers reported feeling they were paid fairly for their job duties (17.0\%). Three-quarters routinely provided FP services $(75.5 \%)$, and nearly two-thirds of providers displayed at least one type of FP bias related to age, parental consent, spousal consent, or parity (62.3\%).

\section{Receipt of FP information}

\section{Descriptive characteristics for receipt of FP information}

About half of clients reported receiving FP information during their non-FP visit (51.7\%). Among those who reported receiving FP information, the provider speaking with the client was the most common source of FP information (98.5\%). The most commonly discussed methods were injection (80.1\%), implant (78.7\%), and intrauterine device (70.6\%) (Table 1). More than half of clients reported wanting to wait for more than two years before having another child (56.6\%), and 19.1\% of clients reported not desiring to have any more children. More than half of PL (60.3\%) and delivery (52.7\%) care clients reported receiving FP information, compared to $45.6 \%$ of well-baby care and $33.3 \%$ of other routine services clients (Fig. 1).

\section{Bivariate analyses}

Results of bivariate analyses for Receipt of FP information are displayed in the Appendix. Client covariates with a significant positive association with receipt of FP information included Client age, PL care was the primary service sought, Highest education attended, Wealth, Number of prior pregnancies, Has had FP discussion with partner, and Heard FP messages in the last 3 months. 
Table 1 Characteristics of Women Included in the Family Planning Integration Study Sample-Kigoma Region, Tanzania, April to July $2016(n=1505)$

\begin{tabular}{lll}
\hline & Women, $\mathrm{n}(\%)$ & $95 \% \mathrm{Cl}$ \\
\hline Age in years & & \\
$15-19$ & $214(14.2)$ & $12.5-16.0$ \\
$20-29$ & $750(49.8)$ & $47.3-52.4$ \\
$30-39$ & $427(28.4)$ & $26.1-30.7$ \\
$40-49$ & $93(6.2)$ & $5.0-7.4$ \\
Don't know & $21(1.4)$ & $0.8-2.0$ \\
Delivery care was primary service sought & & \\
Yes & $935(62.1)$ & $59.7-64.6$ \\
No & $570(37.9)$ & $35.5-40.4$ \\
Well-baby care was primary service sought & & \\
Yes & $272(18.1)$ & $20.5-24.8$ \\
No & $1233(81.9)$ & $79.9-83.8$ \\
Pregnancy loss (PL) care was primary service & & \\
sought & & \\
Yes & $229(15.2)$ & $13.4-17.0$ \\
No & $1276(84.8)$ & $82.9-86.5$
\end{tabular}

Other routine services (postnatal care, HIV testing and management, STI, other) was primary service sought

$\begin{array}{lll}\text { Yes } & 69(4.6) & 3.5-5.6 \\ \text { No } & 1436(95.4) & 94.2-96.4\end{array}$

Facility type

Hospital

Health center

Dispensary

$421(28.0) \quad 25.7-30.2$

$736(48.9) \quad 46.4-51.4$

Highest education attended

No education

Primary

Secondary or higher

$325(21.6) \quad 19.5-23.7$

$990(65.8) \quad 63.4-68.2$

$190(12.6) \quad 10.9-14.3$

Literacy

Can read and write

Cannot read or write

Can either read or write, but not both

$1070(71.1) \quad 68.8-73.4$

$64(4.3) \quad 3.2-5.3$

Missing or refused

$11(0.7) \quad 0.3-1.2$

Wealth

\section{Low wealth \\ Middle wealth}

High wealth

Number of live births

$\begin{array}{lll}\text { None } & 43(2.9) & 2.0-3.7 \\ 1 \text { to } 2 & 662(44.0) & 41.5-46.5 \\ 3 \text { to } 5 & 504(33.5) & 31.1-35.9 \\ 6+ & 293(19.5) & 17.5-21.5 \\ \text { Missing } & 3(0.2) & 0.0-0.4\end{array}$

$\begin{array}{ll}478(31.8) & 29.4-34.1 \\ 514(34.2) & 31.8-36.6\end{array}$

$348(23.1) \quad 21.0-25.3$

$360(23.9) \quad 21.7-26.1$

Table 1 Characteristics of Women Included in the Family Planning Integration Study Sample-Kigoma Region, Tanzania, April to July $2016(n=1505)$ (Continued)

\begin{tabular}{lll}
\hline & Women, $\mathrm{n}(\%)$ & $95 \% \mathrm{Cl}$ \\
\hline Number of prior pregnancies & & \\
0 to 3 & $672(55.3)$ & $52.8-57.8$ \\
$4+$ & & $42.2-47.2$ \\
Marital status & $1361(90.4)$ & $88.8-91.8$ \\
In a union & $144(9.6)$ & $8.2-11.2$ \\
$\quad$ Not in a union & & \\
Has had FP discussion with partner & $894(59.4)$ & $56.9-61.9$ \\
Yes & $603(40.1)$ & $37.6-42.5$ \\
No or don't know & $8(0.5)$ & $0.2-0.9$ \\
Missing & & \\
Able to make own FP decisions & $270(17.9)$ & $16.0-19.9$ \\
Yes & $1231(81.8)$ & $79.8-83.7$ \\
No or don't know & $4(0.3)$ & $0.0-0.5$ \\
Missing & & \\
Received FP information during non-FP visit & & $478(51.7)$ \\
Yes & $727(48.3)$ & $49.2-54.2$ \\
No & $45.8-50.8$
\end{tabular}

Number of FP methods information was received about during non-FP visit

$\begin{array}{lll}0 \text { or } 1 \text { FP method } & 798(53.0) & 50.5-55.5 \\ 2 \text { or more FP methods } & 707(47.0) & 44.5-49.5\end{array}$

Modern FP methods information received about among women who reported receiving information about 1 or more methods $(n=778)^{\mathrm{a}}$

$\begin{array}{lll}\text { Injection } & 623(80.1) & 77.1-82.7 \\ \text { Implant } & 612(78.7) & 75.6-81.4 \\ \text { Intrauterine device } & 549(70.6) & 67.3-73.7 \\ \text { Pills } & 528(67.9) & 64.5-71.1 \\ \text { Male condom } & 262(33.7) & 30.4-37.1 \\ \text { Female condom } & 117(15.0) & 12.7-17.7 \\ \text { Tubal ligation } & 100(12.9) & 10.7-15.4 \\ \text { Vasectomy } & 28(3.6) & 2.5-5.2 \\ \text { Emergency Contraception } & 25(3.2) & 2.2-4.7\end{array}$

Number of sources FP information was received from during non-FP visit

0 or 1 source of FP information

$1349(89.6) \quad 88.0-91.1$

2 or more sources of FP information

$155(10.3)$

$8.9-11.9$

Missing

$1(0.1)$

$0.0-0.5$

Sources that FP information received from among women who reported receiving information from 1 or more sources $(n=777)^{a}$

Provider spoke to client

$766(98.5) \quad 97.3-99.1$

FP educational talk

$89(11.3) \quad 9.3-13.8$

FP educational flyer

$67(8.6) \quad 6.8-10.8$

Client asked provider 
Table 1 Characteristics of Women Included in the Family Planning Integration Study Sample-Kigoma Region, Tanzania, April to July $2016(n=1505)$ (Continued)

\begin{tabular}{|c|c|c|}
\hline & Women, n (\%) & $95 \% \mathrm{Cl}$ \\
\hline FP educational video & $10(1.3)$ & $0.7-2.4$ \\
\hline \multicolumn{3}{|c|}{ Received modern FP method during non-FP visit } \\
\hline No & $1378(91.6)$ & $90.0-92.9$ \\
\hline Yes & $127(8.4)$ & $7.1-10.0$ \\
\hline \multicolumn{3}{|c|}{$\begin{array}{l}\text { Modern FP method received among women who reported } \\
\text { receiving a modern method }(n=127)^{\mathrm{a}}\end{array}$} \\
\hline Injection & $43(33.9)$ & $26.1-42.6$ \\
\hline Implant & $39(30.7)$ & $23.2-39.4$ \\
\hline Male condom & $18(14.2)$ & $9.1-21.5$ \\
\hline Intrauterine device & $14(11.0)$ & $6.6-17.9$ \\
\hline Pill & $9(7.1)$ & $3.7-13.2$ \\
\hline Tubal ligation & $3(2.4)$ & $0.8-7.2$ \\
\hline Female condom & $1(0.8)$ & $0.1-5.5$ \\
\hline \multicolumn{3}{|c|}{ Heard FP messages from any source in last 3 months } \\
\hline Yes & $555(36.9)$ & $34.4-39.3$ \\
\hline No or don't know & $950(63.1)$ & $60.7-65.6$ \\
\hline \multicolumn{3}{|l|}{ Desired timing of future childbearing } \\
\hline $1-2$ years & $287(19.1)$ & $17.8-21.1$ \\
\hline More than 2 years & $852(56.6)$ & $54.1-59.1$ \\
\hline Does not want & $288(19.1)$ & $17.1-21.1$ \\
\hline Don't know or refused & $78(5.2)$ & $4.1-6.3$ \\
\hline \multicolumn{3}{|c|}{ Frequency of attendance at religious services } \\
\hline Attends at least weekly & $1291(85.8)$ & $84.0-87.5$ \\
\hline Attends less often than weekly & $214(14.2)$ & $12.5-16.0$ \\
\hline
\end{tabular}
by some clients

Client covariates with a significant negative association with receipt of FP information included Other routine services was the primary service sought, Frequency of attendance at religious services, and Desired timing of future childbearing.

Provider covariates with a significant positive association with receipt of FP information included Provider highest education completed, Routinely provides FP services, FP Ever-training PCA score, FP Recent-training PCA score, FP Practice PCA score, Perception in-service training has helped job performance, and Access to electronic mentoring opportunities. Hours worked per week was the only provider covariate with a significant negative association with receipt of FP information.

\section{Multilevel analyses}

Results of multilevel, mixed-effects logistic regression analyses for Receipt of FP information are shown in Table 3. The odds of receiving FP information were higher for PL clients vs those seeking any other types of care (aOR 1.97,
95\% CI 1.01-3.84), for those with four or more vs fewer pregnancies (aOR 1.78, 95\% CI 1.21-2.60), and for those who had, vs had not, had a FP discussion with their partner (aOR 1.73, 95\% CI 1.33-2.25).

The odds of receiving FP information were lower for clients aged 40 to 49 years vs those aged 15 to 19 years (aOR 0.50 , 95\% CI $0.26-0.97$ ) and for clients who reported attendance at religious services weekly or more often vs those who reported less frequent attendance (aOR 0.61, 95\% CI $0.44-0.85$ ). Client-level variables found not to have a significant adjusted relationship with receipt of FP information include client Other services was primary service sought, Highest education attended, Wealth, Desired timing of future childbearing, and Heard FP messages in last three months.

The odds of receiving FP information were higher for clients of providers who perceived that in-service training has vs has not helped their job performance (aOR 2.27, 95\% CI 1.35-3.84), and for clients of providers who had high as compared to low FP recent training scores (aOR 1.58, 95\% CI 1.11-2.26). Provider-level variables found not to have a significant adjusted relationship with receipt of FP information include Provider highest education completed, Work hours per week, Routinely provides FP services, FP ever-training $P C A$ score, FP practice PCA score, and Access to electronic mentoring opportunities.

The intraclass correlation (ICC) describes the proportion of the total variance that can attributed to the hierarchal grouping by the provider variable. Net of all predictors included in the final FP information model, about one-quarter of the total variance $(\mathrm{ICC}=0.27$ ) occurred between providers.

\section{Receipt of a modern FP method}

Descriptive characteristics for receipt of a modern FP method Less than one in 10 clients (8.4\%) reported receiving a modern FP method; among those who reported receiving a modern method, injection (33.9\%), implant (30.7\%), and male condoms (14.2\%) were the most common methods received (Table 1). About one-quarter of PL (23.6\%) and 7.3\% of delivery care clients reported receiving a modern method, while only $1.5 \%$ of well-baby care and other routine services clients reported receiving a method (Fig. 1). Sixteen percent (15.7\%) of clients who received information on two or more FP methods reported receiving a modern FP method at their care visit, while only $2.0 \%$ of clients who received information on zero or one method reported this (Fig. 2). Similarly, one-fifth of clients $(20.0 \%)$ who received FP information from two or more sources reported receiving a modern FP method, while only $7.1 \%$ of clients who received FP information from zero or one source reported receiving a method (Fig. 2).

\section{Bivariate analyses}

Results of bivariate analyses for Receipt of a modern FP method are displayed in the Appendix. The client covariates with a significant positive association with receipt of a 
Table 2 Characteristics of Providers included in the Family Planning Integration Study Sample-Kigoma Region, Tanzania, April to July $2016(n=330)$

\begin{tabular}{|c|c|c|}
\hline & Providers, n (\%) & $95 \% \mathrm{Cl}$ \\
\hline \multicolumn{3}{|l|}{ Age in years } \\
\hline $20-29$ & $125(37.9)$ & $32.6-43.1$ \\
\hline $30-39$ & $54(16.4)$ & $12.4-20.4$ \\
\hline $40-49$ & $74(22.4)$ & $17.9-26.9$ \\
\hline $50+$ & $77(23.3)$ & $18.7-27.9$ \\
\hline \multicolumn{3}{|l|}{ Sex } \\
\hline Female & $209(63.3)$ & $58.1-68.6$ \\
\hline Male & $121(36.7)$ & $31.4-41.9$ \\
\hline \multicolumn{3}{|l|}{ Highest education completed } \\
\hline Primary & $15(4.6)$ & $2.3-6.8$ \\
\hline Secondary & $95(28.8)$ & $23.9-33.7$ \\
\hline College/university & $220(66.7)$ & $61.6-71.8$ \\
\hline \multicolumn{3}{|l|}{ Cadre } \\
\hline Clinician & $69(20.9)$ & $16.5-25.3$ \\
\hline Nurse/midwife & $176(53.3)$ & $47.9-58.7$ \\
\hline Other staff & $85(25.7)$ & $21.0-30.5$ \\
\hline \multicolumn{3}{|l|}{ Years in cadre } \\
\hline 0 to 10 years & $127(38.5)$ & $33.2-43.8$ \\
\hline $11+$ years & $203(61.5)$ & $56.2-66.8$ \\
\hline \multicolumn{3}{|l|}{ Years at the facility } \\
\hline 0 to 7 years & $99(30.0)$ & $25.0-35.0$ \\
\hline $8+$ years & $231(70.0)$ & $65.0-75.0$ \\
\hline \multicolumn{3}{|l|}{ Facility type } \\
\hline Hospital & $82(24.9)$ & $20.2-29.5$ \\
\hline Health center & $182(55.2)$ & $49.8-60.5$ \\
\hline Dispensary & $66(20.0)$ & $15.7-24.3$ \\
\hline \multicolumn{3}{|l|}{ Work hours per week } \\
\hline $54+$ hours per week & $191(57.9)$ & $52.5-63.2$ \\
\hline Less than $54 \mathrm{~h}$ per week & $139(42.1)$ & $36.8-47.5$ \\
\hline \multicolumn{3}{|l|}{ Routinely provides FP services } \\
\hline Yes & $249(75.5)$ & $70.8-80.1$ \\
\hline No & $81(24.6)$ & $19.9-29.2$ \\
\hline \multicolumn{3}{|l|}{ FP ever-training PCA score ${ }^{a}$} \\
\hline Low & $132(40.0)$ & $34.7-45.3$ \\
\hline High & $198(60.0)$ & $54.7-65.3$ \\
\hline \multicolumn{3}{|l|}{ FP recent-training PCA Score ${ }^{a}$} \\
\hline Low & $227(68.8)$ & $63.8-73.8$ \\
\hline High & $103(31.2)$ & $26.2-36.2$ \\
\hline \multicolumn{3}{|l|}{ FP Practice PCA Score ${ }^{a}$} \\
\hline Low & $131(39.7)$ & $34.4-45.0$ \\
\hline High & $199(60.3)$ & $55.0-65.6$ \\
\hline
\end{tabular}

Table 2 Characteristics of Providers included in the Family Planning Integration Study Sample-Kigoma Region, Tanzania, April to July $2016(n=330)$ (Continued)

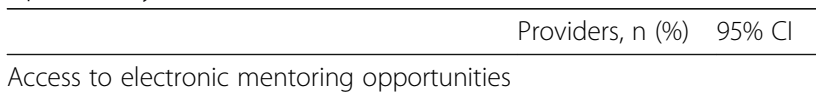

$\begin{array}{lll}\text { No access } & 165(50.0) & 44.6-55.4 \\ \text { Access to at least } 1 \text { type (emergency call } & 165(50.0) & 44.6-55.4\end{array}$
system, e-learning, teleconference)

Perception in-service training has helped job performance

$\begin{array}{lll}\text { Yes } & 285(86.4) & 82.6-90.1 \\ \text { No } & 45(13.6) & 9.9-17.4\end{array}$

Job Satisfaction

$\begin{array}{lll}\text { Very or a little satisfied } & 148(44.9) & 39.5-50.2 \\ \text { Neutral, a little dissatisfied or very } & 182(55.2) & 49.8-60.5\end{array}$
dissatisfied

Perception paid fairly for job duties

$\begin{array}{lll}\text { Yes } & 56(17.0) & 12.9-21.0 \\ \text { No } & 274(83.0) & 79.0-87.1\end{array}$

Perception of adequacy of training for job duties

$\begin{array}{lll}\text { Yes } & 227(68.8) & 63.8-73.8 \\ \text { No } & 103(31.2) & 26.2-36.2\end{array}$

Displays at least $1 \mathrm{FP}$ bias $^{\mathrm{b}}$

$\begin{array}{lll}\text { Yes } & 207(62.7) & 57.5-68.0\end{array}$

No $\quad 123$ (37.3) 32.0-42.5

aproviders were asked if they had ever received training in, had recently received training in (in the last 18 months), and if they had provided the service in the last 3 months related to the following 14 FP items: 1) counsel women about FP and contraception; 2) give an injectable; 3) insert an IU(C)D; 4) remove an IU(C)D; 5) insert an implant; 6) remove an implant; 7) perform a tubal ligation; 8) perform a vasectomy; 9) provide counseling on FP options; 10) provide counseling on the efficacy of FP methods; 11) provide counseling on the potential side effects of FP methods; 12) provide counseling on the potential warning signs of FP methods; 13) provide clinical management of FP side effects; and 14) provide FP for HIV positive women

bProviders were asked if they decide whether or not to provide FP services to clients based on one or more of the following client characteristics: marital status/parental consent/partner consent/age/parity

modern contraceptive method included PL care was the primary service sought, Client highest education attended, Literacy, Wealth, Number of live births, Has had FP discussion with partner, Able to make own FP decisions, Number of FP methods received information about, and Number of sources for FP information. Client covariates with a significant negative association with receipt of a modern contraceptive method included Well-baby care was the primary service sought, Other routine services was the primary service sought, Marital status, and Desired timing of future childbearing.

Provider covariates with a significant positive association with receipt of a modern contraceptive method included FP Practice PCA score and Access to electronic mentoring opportunities. Provider covariates with a significant negative 


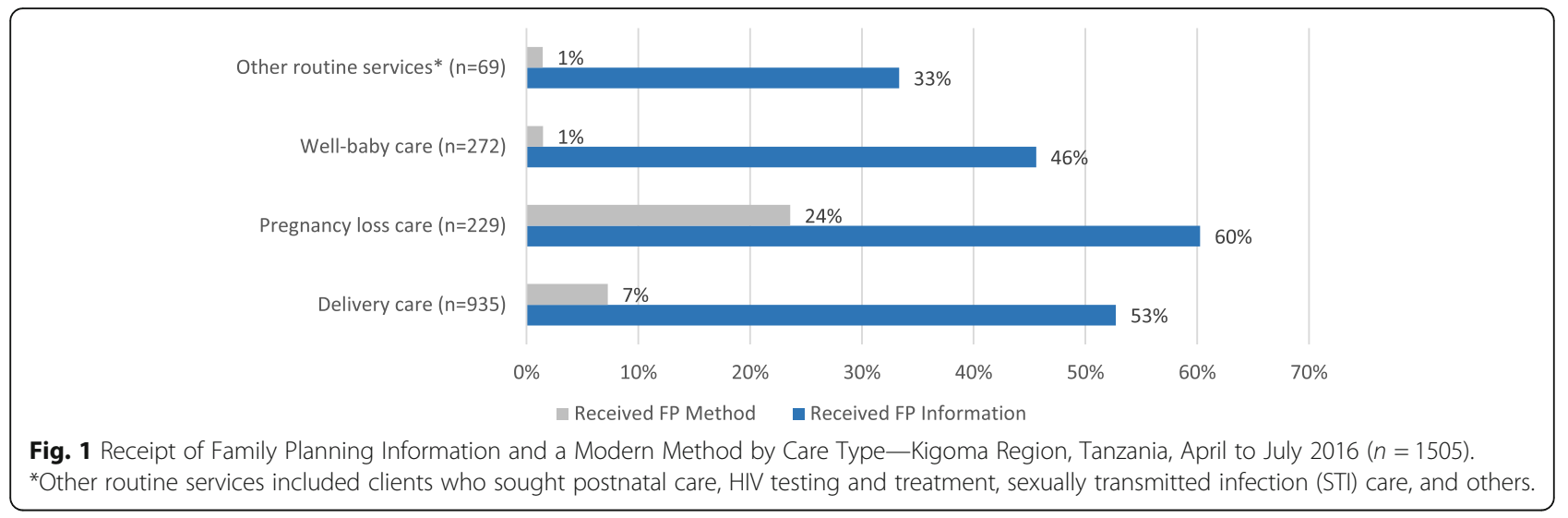

association with receipt of a modern contraceptive method included Provider age, Cadre, and Work hours per week.

\section{Multilevel analyses}

Results of multilevel, mixed-effects logistic regression analyses for receipt of a modern FP method are shown in Table 4. The odds of receiving a modern FP method were higher for clients who received information on two or more vs fewer methods (aOR 7.13, 95\% CI 2.13-23.80), for those who had a FP discussion with their partner (aOR 5.87, 95\% CI 2.81-12.28), for clients seeking PL care vs those seeking all other types of care (aOR 4.08, 95\% CI 1.97-8.44), for those with zero vs one or more live births (aOR 3.92, 95\% CI 1.40-10.94), for those who reported they are able to make their own FP decisions (aOR 3.17, 95\% CI 1.55-6.49), for clients who received FP information from two or more vs fewer sources (aOR 3.12, 95\% CI 1.40-6.92), and for those in the middle and high wealth terciles vs low wealth tercile (aOR 1.99, 95\% CI 1.11-3.56; aOR 2.30, 95\% CI 1.30-4.07, respectively).

Well-baby care clients, Other services clients, and married clients had significantly lower odds of receiving a FP method compared to clients seeking any other type of services and unmarried clients (aOR 0.14, 95\% CI 0.04-0.54; aOR 0.08, 95\% CI 0.01-0.87; aOR 0.41, 95\% CI 0.18-0.93, respectively). Client-level variables found not to have a significant adjusted relationship with receipt of a FP method include Highest education attended, Literacy, and Desired timing of future childbearing.

None of the provider-level variables were found to have a significant adjusted relationship with receipt of a modern FP method by a client. Net of all predictor variables included in the FP method model, nearly $30 \%$ of the total variance (ICC 0.30 ) occurred between providers.

\section{Discussion}

Integrating FP service provision into other types of healthcare visits can provide an important opportunity to reduce the unmet need for FP and ultimately lower maternal and neonatal mortality. We sought to understand the factors that predicted receipt of FP information and a modern FP method among women attending maternal, child, and reproductive health care services in Kigoma, Tanzania. The results demonstrate the association between the receipt of FP information with both the client and provider characteristics, while receipt of a modern FP method is associated with only client factors. This information will help inform FP training and programming in Kigoma, Tanzania.

First, level of FP integration is not consistent across non-FP care types. Unlike results from a study in Senegal, which found low receipt of FP information among PL clients compared to clients of other care types [16], our study found that women who sought PL services had nearly two times greater odds of receiving FP information and more than four times greater odds of receiving a modern method compared to women seeking other types of services. In contrast, women who sought well-baby care services and other routine care services had 86 and 92\% lower odds of receiving a modern FP method, respectively.

Women's self-efficacy, assessed with questions about past FP discussions with partner and ability to make one's own FP decisions, was strongly associated with receipt of information and modern FP methods. Women who reported past FP discussions with their partner as compared with those who did not had nearly two times greater odds of receiving FP information and more than five times greater odds of receiving a FP method. In addition, women who reported the ability to make their own FP decisions had three times greater odds of receiving a method compared to women unable to make their own decisions. These results align with previous studies in India, Senegal, and Turkey, which also found that that partner communication $[16,25]$ and self-efficacy $[20]$ are positively associated with FP uptake. These findings suggest that women who have had conversations with their FP partners and/or feel they can make their own decisions may be initiating 
Table 3 Multilevel Mixed-Effects Logistic Regression Analysis for Receipt of Family Planning (FP) Information-Kigoma Region, Tanzania, April to July 2016 (Clients $n=1497$, Providers $n=330$ )

\begin{tabular}{lll}
\hline & $\begin{array}{l}\text { Adjusted OR } \\
(95 \% \mathrm{Cl})\end{array}$ & $p$-value \\
\hline FIXED EFFECTS - CLIENT LEVEL VARIABLES & & \\
Client age in years & & \\
15-19 (reference) & & \\
$20-29$ & $0.93(0.66-1.33)$ & 0.707 \\
$30-39$ & $0.61(0.37-1.03)$ & 0.063 \\
40-49 & $0.50(0.26-0.97)$ & 0.039 \\
Don't know & $4.11(0.95-17.76)$ & 0.058 \\
Highest education attended & & \\
No education & & \\
Primary & $1.25(0.87-1.79)$ & 0.230 \\
Secondary & $1.53(0.93-2.51)$ & 0.093 \\
College or university & $1.03(0.39-2.74)$ & 0.947
\end{tabular}

Pregnancy loss (PL) care was primary service sought

No (reference)

Yes

$1.97(1.01-3.84)$

Other routine services was primary service sought

No (reference)

Yes

$0.43(0.18-1.03)$

Frequency of religious service attendance

Less often than weekly (reference)

Weekly or more often

$0.61(0.44-0.85)$

0.003

Wealth

Low wealth (reference)

Middle wealth

$1.24(0.82-1.88) \quad 0.300$

High wealth

$1.27(0.81-1.97) \quad 0.294$

Number of prior pregnancies

0 to 3 (reference)

$4+$

$1.78(1.21-2.60)$

0.003

Desired timing of future childbearing

More than 2 years, or does not want (reference)

1 to 2 years

$0.73(0.51-1.04)$

Heard FP messages in last three months

No (reference)

Yes

$1.21(0.88-1.67)$

Has had FP discussion with partner

No or don't know (reference)

Yes

$1.73(1.33-2.25)$

0.000

FIXED EFFECTS - PROVIDER-LEVEL VARIABLES

Highest education completed

Primary (reference)

Secondary

$1.25(0.60-2.63)$
Table 3 Multilevel Mixed-Effects Logistic Regression Analysis for Receipt of Family Planning (FP) Information-Kigoma Region, Tanzania, April to July 2016 (Clients $n=1497$, Providers $n=330$ ) (Continued)

\begin{tabular}{|c|c|c|}
\hline & $\begin{array}{l}\text { Adjusted OR } \\
(95 \% \mathrm{Cl})\end{array}$ & $p$-value \\
\hline College/University & $2.03(0.97-4.25)$ & 0.060 \\
\hline \multicolumn{3}{|l|}{ Work hours per week } \\
\hline \multicolumn{3}{|c|}{ Less than $54 \mathrm{~h}$ per week (reference) } \\
\hline $54+$ hours per week & $0.68(0.45-1.01)$ & 0.054 \\
\hline \multicolumn{3}{|c|}{ Routinely provides FP services } \\
\hline \multicolumn{3}{|l|}{ No (reference) } \\
\hline Yes & $1.45(0.85-2.47)$ & 0.170 \\
\hline \multicolumn{3}{|c|}{ FP ever-training PCA score ${ }^{a}$} \\
\hline \multicolumn{3}{|l|}{ Low (reference) } \\
\hline High & $1.01(0.57-1.79)$ & 0.970 \\
\hline \multicolumn{3}{|c|}{ FP recent-training PCA score ${ }^{a}$} \\
\hline \multicolumn{3}{|l|}{ Low (reference) } \\
\hline High & $1.58(1.11-2.26)$ & 0.012 \\
\hline \multicolumn{3}{|l|}{ FP Practice PCA Score ${ }^{a}$} \\
\hline \multicolumn{3}{|l|}{ Low (reference) } \\
\hline High & $0.86(0.48-1.53)$ & 0.606 \\
\hline
\end{tabular}

Access to electronic mentoring opportunities

No access (reference)

Access to at least 1 type (emergency $1.38(0.85-2.23) \quad 0.191$ call system, e-learning, teleconference)

Perception in-service training has helped job performance

No (reference)

Yes

$2.27(1.35-3.84)$

0.002

RANDOM EFFECTS

Provider-level variance (SE)

$1.21(0.28)$

Provider-level variance

$0.27(0.19-0.37)$

partition coefficient

Level 1 units

1497

Level 2 units

330

Log likelihood

$-921.18412$

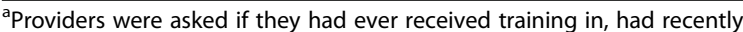
received training in (in the last 18 months), and if they had provided the service in the last 3 months related to the following 14 FP items: 1) counsel women about FP and contraception; 2) give an injectable; 3 ) insert an IU(C)D; 4) remove an IU(C)D; 5) insert an implant; 6) remove an implant; 7) perform a tubal ligation; 8) perform a vasectomy; 9) provide counseling on FP options; 10) provide counseling on the efficacy of FP methods; 11) provide counseling on the potential side effects of FP methods; 12) provide counseling on the potential warning signs of FP methods; 13) provide clinical management of FP side effects; and 14) provide FP for HIV positive women

FP conversations with providers, thereby driving the receipt of information and a method during a care visit.

The quantity of FP information received at the visit, including the number of methods information was received about and the number of sources information came from, 


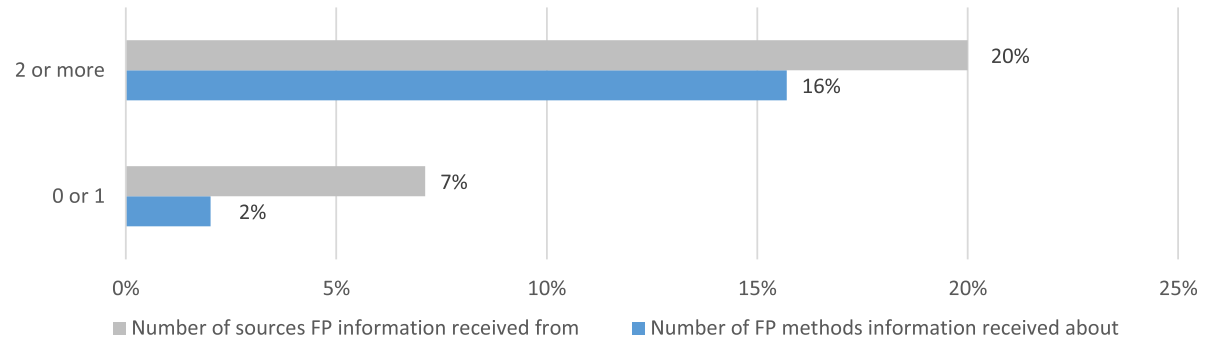

Fig. 2 Percentage of Clients Who Received a Modern Family Planning Method by Number of Methods Information was Received about and Number of Sources Information was Received from—Kigoma Region, Tanzania, April to July 2016 ( $n=1505)$

was strongly associated with FP method receipt. Women who received FP information about two or more methods had seven times greater odds of receiving a contraceptive method compared to women who received FP information about one or zero methods. Similarly, women who received FP information from two or more sources had nearly three times greater odds of receiving a method compared to women who received FP information from one or zero sources. These findings may inform elements of counseling in clinical practice.

Additional demographic characteristics, including parity, marital status, religiosity, and wealth, were also associated with receipt of FP information and modern FP methods. Women with four or more live births had nearly two times greater odds of receiving FP information compared to women with three or fewer live births. One possible explanation for this is providers' perceptions of women's eligibility for receiving methods. A study on provider-imposed barriers to family planning in Kenya found that women's parity influenced providers' method offerings; specifically, providers reported to impose FP method restrictions on women without children [7]. However, we found that women reporting having no children born alive had nearly four times greater odds of receiving a FP method. This finding may represent a desire to postpone childbearing. Specifically, among this sub-group of clients with no live births, a comparatively higher proportion were teenagers $(38.1 \%$ vs $13.6 \%)$, unwed (25.6\% vs $8.9 \%)$, and seeking PL care $(93.0 \%$ vs $13.0 \%$ ) compared to those with live births. We also found that women with no live births were not more likely to receive FP information, suggesting that this sub-group of clients were driving the requests for receipt of FP methods.

Women who reported more frequent attendance at religious services had 39\% lower odds of receiving FP information compared to women with less frequent attendance. This finding suggests that religiosity either influences women's request for FP information or influences provider's perception of client interest in FP. Women in the middle and high wealth categories had two and two and a half times greater odds of receiving a FP method, respectively. This finding aligns with previous findings that poorer women receiving FP counseling and education have lower uptake of FP methods [12]. Given that FP is available at no cost to women in Tanzania, this difference may reflect an inverse relationship between future fertility preferences and wealth. Alternatively, this finding might suggest a provider's bias in offering modern methods to wealthier clients.

Recent in-service training and positive perceptions of in-service training as a whole among providers were important factors for client receipt of FP information. Other studies have found that a greater proportion of providers without in-service FP training imposed parity, age, marital, and other restrictions on provision of FP methods [7, 29, 36, 37]. In our study, clients of providers who had recent in-service training on a higher number of FP topics had one and a half times greater odds of receiving FP information at their visit compared to clients of providers who had recent in-service training on a lower number of FP topics. Similarly, clients of providers who reported that in-service training has helped their job performance had more than two times greater odds of receiving FP information compared clients of providers who reported in-service training has not helped. While we found that provider factors were important for client's receipt of FP information, provider factors were not predictive of client's receipt of a FP method. This suggests that client's desire for adopting a modern method expressed during non-FP visits may be the primary driver of receiving FP methods.

Findings from this study corroborate evidence that women's self-efficacy in FP decision-making [20], women's specific demographic characteristics (e.g., religiosity, wealth) [12], and providers' in-service training [7, 29] influence women's odds of receiving a FP method. This study adds new evidence pertaining to important elements of FP counseling and receipt of a method-namely, the quantity of FP information received at a single care visit (e.g., number of methods described, number of sources of FP information). While many studies address factors associated with receipt of FP methods, this study also adds new insight into factors associated with receipt of FP information, including provider characteristics related to the timing $\mathrm{FP}$ in-service training, partner communication, high parity, older age, and lack of religiosity. 
Table 4 Multilevel Mixed Effects Logistic Regression Analysis for Receipt of a Modern Family Planning (FP) Method-Kigoma Region, Tanzania, April to July 2016 (Clients $n=1482$, Providers $n=330$ )

\begin{tabular}{ll}
\hline & $\begin{array}{l}\text { Adjusted OR } \\
(95 \% \mathrm{Cl})\end{array}$ \\
\end{tabular}

FIXED EFFECTS - CLIENT LEVEL VARIABLES

Highest education attended

No education (reference)

Primary

Secondary or higher

University

$0.43(0.17-1.13)$

0.088

$0.85(0.24-3.03) \quad 0.799$

$1.39(0.16-11.87) \quad 0.765$

Literacy

Cannot read or write, or can do

one (reference)

read and write

$1.89(0.72-4.91)$

0.193

Pregnancy loss (PL) care was primary service sought

No (reference)

Yes

$4.08(1.97-8.44)$

0.000

Other services was primary service sought

No (reference)

Yes

$0.08(0.01-0.87)$

0.038

Well-baby care was primary service sought

No (reference)

Yes

$0.14(0.04-0.54)$

0.004

Marital status

Not in a union (reference)

In a union

$0.41(0.18-0.93)$

0.034

Wealth

Low wealth (reference)

Middle wealth

$1.99(1.11-3.56)$

0.021

Highest wealth

$2.30(1.30-4.07)$

0.004

Live births

1 or more (reference)

None

$3.92(1.40-10.94)$

0.009

Has had FP discussion with partner

No or don't know (reference)

Yes

$5.87(2.81-12.28)$

Can make own FP decisions

No or don't know (reference)

Yes

$3.17(1.55-6.49)$

0.002

Number of FP methods received information about during non-FP visit

0 to 1 (reference)

2 or more

$7.13(2.13-23.80)$
Table 4 Multilevel Mixed Effects Logistic Regression Analysis for Receipt of a Modern Family Planning (FP) Method-Kigoma Region, Tanzania, April to July 2016 (Clients $n=1482$, Providers $n=330$ ) (Continued)

\begin{tabular}{|c|c|c|}
\hline & $\begin{array}{l}\text { Adjusted OR } \\
(95 \% \mathrm{Cl})\end{array}$ & $\overline{p \text {-value }}$ \\
\hline \multicolumn{3}{|l|}{$\begin{array}{l}\text { Number of sources FP information } \\
\text { received from during non-FP visit }\end{array}$} \\
\hline \multicolumn{3}{|l|}{0 to 1 (reference) } \\
\hline 2 or more & $3.12(1.40-6.92)$ & 0.005 \\
\hline \multicolumn{3}{|l|}{ Desired timing of future childbearing } \\
\hline \multicolumn{3}{|l|}{$\begin{array}{l}\text { More than } 2 \text { years or does not want } \\
\text { (reference) }\end{array}$} \\
\hline $1-2$ years & $0.47(0.20-1.13)$ & 0.090 \\
\hline \multicolumn{3}{|l|}{ FIXED EFFECTS - PROVIDER-LEVEL VARIABLES } \\
\hline \multicolumn{3}{|l|}{ Age in years } \\
\hline \multicolumn{3}{|l|}{38 years or younger (reference) } \\
\hline $39+$ years & $0.77(0.37-1.62)$ & 0.498 \\
\hline \multicolumn{3}{|l|}{ Cadre } \\
\hline \multicolumn{3}{|l|}{ Clinician (reference) } \\
\hline Nurse/midwife & $0.81(0.41-1.59)$ & 0.535 \\
\hline Other staff & $0.90(0.35-2.30)$ & 0.824 \\
\hline \multicolumn{3}{|l|}{ Hours worked per week } \\
\hline \multicolumn{3}{|l|}{55 h or less per week (reference) } \\
\hline $56+$ hours & $0.63(0.35-1.15)$ & 0.132 \\
\hline \multicolumn{3}{|l|}{ Access to electronic mentoring opportunities } \\
\hline \multicolumn{3}{|l|}{ No access (reference) } \\
\hline $\begin{array}{l}\text { Access to at least } 1 \text { type (emergency } \\
\text { call system, e-learning, teleconference) }\end{array}$ & $1.27(0.57-2.82)$ & 0.562 \\
\hline \multicolumn{3}{|l|}{ FP Practice PCA Score ${ }^{a}$} \\
\hline \multicolumn{3}{|l|}{ Low (reference) } \\
\hline High & $1.14(0.60-2.17)$ & 0.685 \\
\hline \multicolumn{3}{|l|}{ RANDOM EFFECTS } \\
\hline Provider-level variance (SE) & $1.39(0.71)$ & \\
\hline $\begin{array}{l}\text { Provider-level variance partition } \\
\text { coefficient }\end{array}$ & $0.30(0.13-0.53)$ & \\
\hline Level 1 units & 1482 & \\
\hline Level 2 units & 330 & \\
\hline Log likelihood & -292.07447 & \\
\hline
\end{tabular}

Providers were asked if they had provided the service in the last 3 months related to the following $14 \mathrm{FP}$ items: 1 ) counsel women about FP and contraception; 2) give an injectable; 3) insert an IU(C)D; 4) remove an IU(C)D; 5) insert an implant; 6) remove an implant; 7) perform a tubal ligation; 8) perform a vasectomy; 9) provide counseling on FP options; 10) provide counseling on the efficacy of FP methods; 11) provide counseling on the potential side effects of FP methods; 12) provide counseling on the potential warning signs of FP methods; 13) provide clinical management of FP side effects; and 14) provide FP for HIV positive women

\section{Strengths and limitations}

This study has several strengths. First, the study has matched client and provider data, enabling quantification of the importance of both client and provider level factors for FP 
integration. In addition, clients of varied care services were included in the analyses, allowing a better understanding of the strengths and limitations of FP integration by care type. Moreover, we operationalized select independent variables in ways that have not been done in previously published work. For example in the model for Receipt of a modern FP method, receipt of FP information was operationalized into two distinct independent variables that quantify same-visit access to information: 1) Number of FP methods information was received about, and 2) Number of sources information received from. Additionally, provider-level FP ever-training score, FP recent-training score, and FP Practice score variables were operationalized using multiple FP care elements to differentiate the importance of both dose and timing of training and practice to provision of FP integration services.

There are several limitations to this study that are important to consider. First, the study employed a cross-sectional design which limits our ability to make causal inferences between the independent variables and outcomes of interest. We noted wide confidence intervals for some variables in the regression analyses which likely reflect small cell sizes; these results should be interpreted with caution. Ongoing longitudinal data collection and analyses will strengthen the robustness of the potential relationships. We used convenience sampling to recruit providers and clients, limiting the generalizability of the findings within or beyond Kigoma Region. To minimize this limitation, we aimed to interview all providers and clients who met the inclusion criteria and were available for interview during the study period. Data limitations included lack of a rural versus urban variable and information about receipt of FP counselling at multiple points in time. These exclusions eliminated our ability to assess the influence of setting and counselling over time on receipt of FP information and uptake.

\section{Research, programmatic, and policy implications}

Our findings highlight areas for future research and have clear implications for programmatic and policy work. Additional research with matched client and provider data is needed to further delineate the relative importance of client and provider factors for FP integration. Studies such as this could be replicated in other areas of Tanzania and eastern Africa, or beyond, to better understand how similar or disparate the determinants of receiving FP information and methods are across regions and countries. Future analyses would benefit from inclusion of interaction terms to better understand how specific client characteristics, such religion, age, and parity, intersect with provider perceptions and potential biases in ways that influence provision of FP services. Observational and descriptive studies are also needed to better understand logistical considerations for integration of services in facilities (e.g., location of FP providers and methods, process for receiving services either directly or through referral, other access barriers).
Increased attention is needed to better integrate FP counseling and service provision routinely into well-baby and other routine care services. Capitalizing on the year post delivery is one strategy that has been effective in other settings $[15,17,23]$. Community- and facility-based strategies are needed that encourage women to have conversations with their partners and to create service environments where women feel they can begin conversations with providers and make their own decisions to initiate FP. Based on our findings, expanding the number of FP method options and sources of information provided at single visits would also likely drive an uptake in FP use. The US-based National Campaign to Prevent Teen and Unplanned Pregnancy's One Key Question is a program that encourages primary care providers to routinely ask women about their reproductive health goals using a simple question: would you like to become pregnant in the next year? [38] This approach, among others, could be piloted in Kigoma, and if deemed effective, incorporated into national policies.

\section{Conclusion}

Despite much evidence establishing the salient role of FP counseling to improve reproductive health outcomes, there is less consensus about the relative impact of client or provider attributes on the receipt of FP information or a modern method. We address the question of whether including both client and provider characteristics adds predictive value when analyzing the risk of FP integration or uptake. Our findings demonstrate that both client and provider factors significantly influence FP integration. Improved understanding of the interplay between clients and providers is critical for the effective provision of FP services and should be considered in the design of reproductive health policies and future data collection exercises.

\section{Endnotes}

${ }^{1}$ Providers were asked if they decide whether or not to provide FP services to clients based on one or more of the following client characteristics: marital status/parental consent/partner consent/age/parity

${ }^{2}$ Providers were asked if they had ever received training in, had recently received training in (in the last 18 months), and if they had provided the service in the last 3 months related to the following 14 FP items: 1) counsel women about FP and contraception; 2) give an injectable; 3) insert an IU(C)D; 4) remove an IU(C)D; 5) insert an implant; 6) remove an implant; 7) perform a tubal ligation; 8) perform a vasectomy; 9) provide counseling on FP options; 10) provide counseling on the efficacy of FP methods; 11) provide counseling on the potential side effects of FP methods; 12) provide counseling on the potential warning signs of FP methods; 13) provide clinical management of FP side effects; and 14) provide FP for HIV positive women. 


\section{Appendix}

Table 5 Association between Client and Provider Characteristics and Receipt of Family Planning (FP) Information and a Modern FP Method_Kigoma Region, Tanzania, April to July 2016 (Clients $n=1505$, Providers $n=330$ )

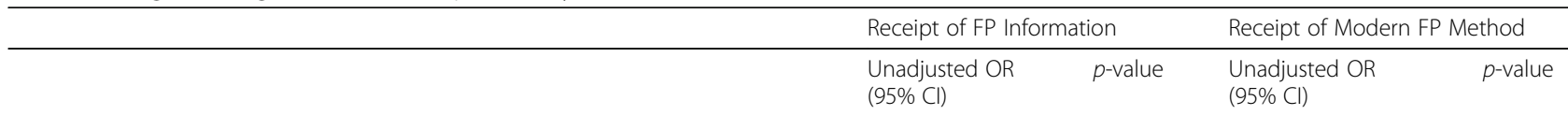

CLIENT LEVEL VARIABLES

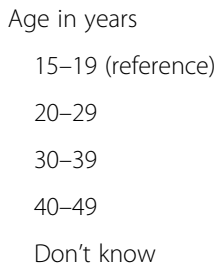


Table 5 Association between Client and Provider Characteristics and Receipt of Family Planning (FP) Information and a Modern FP Method_Kigoma Region, Tanzania, April to July 2016 (Clients $n=1505$, Providers $n=330$ ) (Continued)

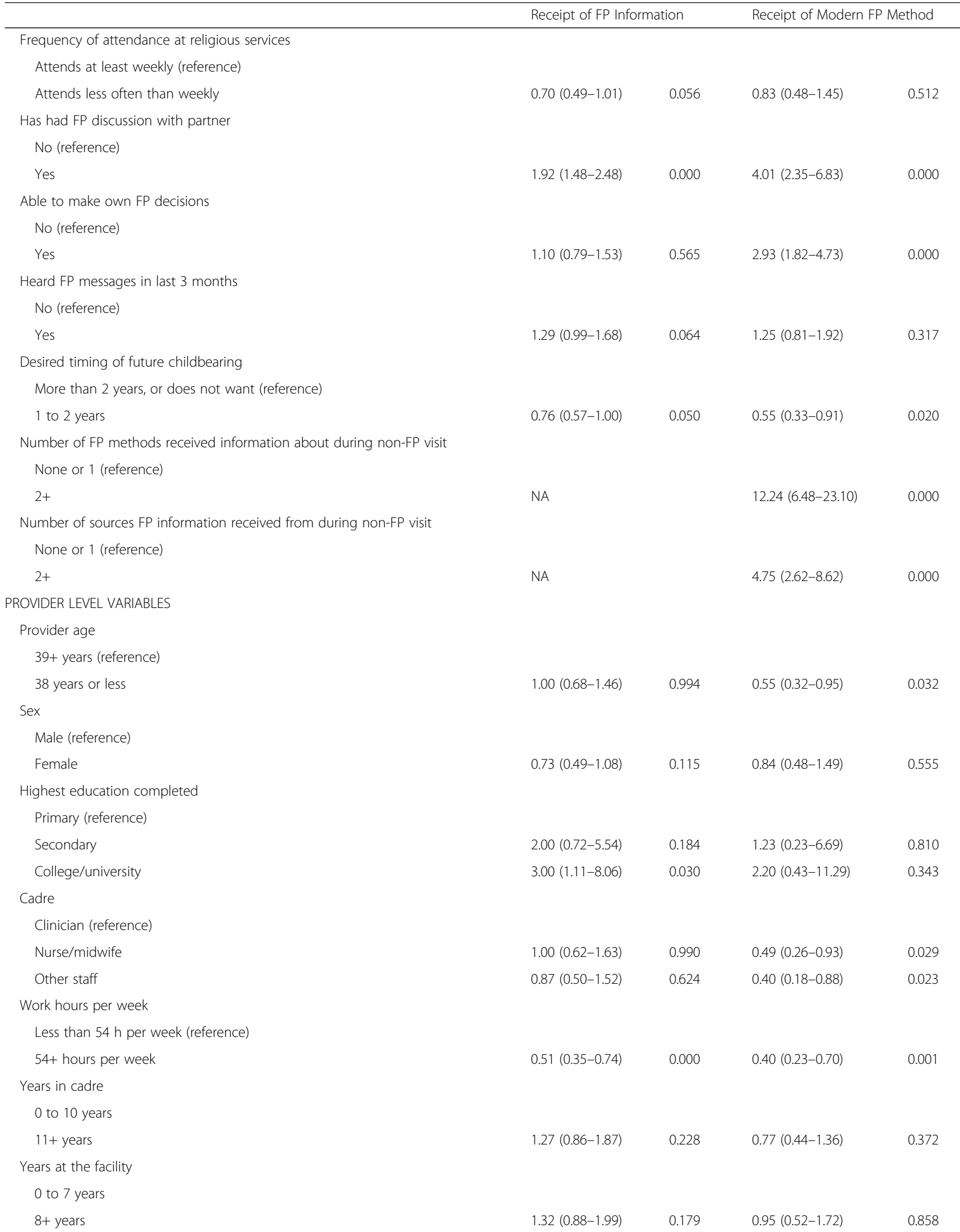


Table 5 Association between Client and Provider Characteristics and Receipt of Family Planning (FP) Information and a Modern FP Method_Kigoma Region, Tanzania, April to July 2016 (Clients $n=1505$, Providers $n=330$ ) (Continued)

\begin{tabular}{|c|c|c|c|c|}
\hline & \multicolumn{2}{|c|}{ Receipt of FP Information } & \multicolumn{2}{|c|}{ Receipt of Modern FP Method } \\
\hline \multicolumn{5}{|l|}{ Routinely provides FP services } \\
\hline \multicolumn{5}{|l|}{ No (reference) } \\
\hline Yes & $2.02(1.30-3.13)$ & 0.002 & $1.19(0.61-2.33)$ & 0.614 \\
\hline \multicolumn{5}{|l|}{ FP ever-training PCA score ${ }^{a}$} \\
\hline \multicolumn{5}{|l|}{ Low (reference) } \\
\hline High & $1.70(1.16-2.49)$ & 0.007 & $1.53(0.85-2.74)$ & 0.153 \\
\hline \multicolumn{5}{|l|}{ FP recent-training PCA score ${ }^{a}$} \\
\hline \multicolumn{5}{|l|}{ Low (reference) } \\
\hline High & $1.70(1.14-2.53)$ & 0.009 & $1.28(0.71-2.28)$ & 0.412 \\
\hline \multicolumn{5}{|l|}{ FP Practice PCA Score ${ }^{a}$} \\
\hline \multicolumn{5}{|l|}{ Low (reference) } \\
\hline High & $1.64(1.11-2.41)$ & 0.013 & $1.72(0.95-3.09)$ & 0.071 \\
\hline \multicolumn{5}{|l|}{ Perception in-service training has helped job performance } \\
\hline \multicolumn{5}{|l|}{ No (reference) } \\
\hline Yes & $2.64(1.51-4.63)$ & 0.001 & $1.75(0.70-4.38)$ & 0.230 \\
\hline \multicolumn{5}{|l|}{ Access to electronic mentoring opportunities } \\
\hline \multicolumn{5}{|l|}{ No access (reference) } \\
\hline Access to at least 1 type (emergency call system, e-learning, teleconference) & $1.88(1.29-0.74)$ & 0.001 & $2.28(1.31-3.98)$ & 0.004 \\
\hline \multicolumn{5}{|l|}{ Job Satisfaction } \\
\hline \multicolumn{5}{|l|}{ Very or a little satisfied (reference) } \\
\hline Neutral or a little or very dissatisfied & $0.95(0.65-1.38)$ & 0.779 & $0.94(0.54-1.64)$ & 0.825 \\
\hline \multicolumn{5}{|l|}{ Perception of adequacy of training for job duties } \\
\hline \multicolumn{5}{|l|}{ No (reference) } \\
\hline Yes & $1.06(0.70-1.60)$ & 0.780 & $1.31(0.70-2.43)$ & 0.402 \\
\hline \multicolumn{5}{|l|}{ Perception in-service training has helped job performance } \\
\hline \multicolumn{5}{|l|}{ No (reference) } \\
\hline Yes & $2.64(1.51-4.63)$ & 0.001 & $1.75(0.70-4.38)$ & 0.230 \\
\hline \multicolumn{5}{|l|}{ Perception paid fairly for job duties } \\
\hline \multicolumn{5}{|l|}{ No (reference) } \\
\hline Yes & $1.43(0.87-2.36)$ & 0.160 & $0.73(0.33-1.58)$ & 0.421 \\
\hline \multicolumn{5}{|l|}{ Displays at least one FP bias ${ }^{b}$} \\
\hline \multicolumn{5}{|l|}{ No (reference) } \\
\hline Yes & $1.16(0.78-1.71)$ & 0.468 & $0.90(0.51-1.59)$ & 0.709 \\
\hline
\end{tabular}

aproviders were asked if they had ever received training in, had recently received training in (in the last 18 months), and if they had provided the service in the last 3 months related to the following 14 FP items: 1) counsel women about FP and contraception; 2) give an injectable; 3) insert an IU(C)D; 4) remove an IU(C)D; 5) insert an implant; 6) remove an implant; 7) perform a tubal ligation; 8) perform a vasectomy; 9) provide counseling on FP options; 10) provide counseling on the efficacy of FP methods; 11) provide counseling on the potential side effects of FP methods; 12) provide counseling on the potential warning signs of FP methods; 13) provide clinical management of FP side effects; and 14) provide FP for HIV positive women

${ }^{b}$ Providers were asked if they decide whether or not to provide FP services to clients based on one or more of the following client characteristics: marital status/parental consent/partner consent/age/parity

\section{Abbreviations}

95\% Cl: 95\% Confidence intervals; ANC: Antenatal care; BEmONC: Basic Emergency Obstetric and Neonatal Care; BRN: Big Results Now; CDC: Centers for Disease Control and Prevention; CEmONC: Comprehensive Emergency Obstetric and Neonatal Care; DRH: Division of Reproductive Health; FP: Family planning; ICC: Intraclass correlation; IUD: Intrauterine Device; LAM: Lactational amenorrhea method; MOHCDGEC: Ministry of Health, Community Development, Gender, the Elderly, and Children; NBS: National
Bureau of Statistics; OR: Odds Ratios; PCA: Principal Components Analysis; PL: Pregnancy loss; PNC: Postnatal Care; TFR: Total Fertility Rate; WHO: World Health Organization

\section{Acknowledgements}

Our sincere gratitude to the clients and providers who so generously gave us their time and shared their experiences. We would also like to thank the 
Kigoma Regional Ministry of Health, AMCA Inter Consult, interviewers, and data entry staff for their dedication to this project. The authors would also like to acknowledge the support of CDC colleagues Susanna Binzen, Fernando Carlosama, Jonetta Mpofu, Michelle Schmitz, and intern from Emory University Rollins School of Public Health, Hannah Nguyen.

\section{Funding}

This study was funded by Bloomberg Philanthropies and the Fondation H\&B Agerup.

\section{Availability of data and materials}

Upon request

\section{Disclaimer}

The findings and conclusions in this report are those of the authors and do not necessarily represent the official position of the US Centers for Disease Control and Prevention.

\section{Declarations}

The data collection for our project was approved by the National Institute for Medical Research (NIMR) in Tanzania as one activity among a larger set of activities for the Project to Reduce Maternal Deaths in Tanzania. Interviewers received one week of training in quantitative interview techniques and best practices in research and ethics. Informed consent was obtained from each participant and confirmed with the participant's thumbprint.

\section{Authors' contributions}

MD was responsible for the study design, undertook the fieldwork and data collection, analysis, interpretation, and helped write the manuscript. EB and DM were involved in the literature search, interpretation of findings, and helped write the manuscript. AR supervised data entry, participated in data cleaning, and helped finalize the manuscript. LK participated in data analysis, interpretation of findings, and helped write the manuscript. WM and PC helped interpret findings. RB helped collect data and interpret findings. FS helped with the study design and interpretation of findings. All authors read and approved the final manuscript submitted for publication.

\section{Consent for publication}

Not applicable.

\section{Competing interests}

The authors have no competing interests to declare.

\section{Publisher's Note}

Springer Nature remains neutral with regard to jurisdictional claims in published maps and institutional affiliations.

\section{Author details}

${ }^{1}$ Centers for Disease Control and Prevention, Division of Reproductive Health, Atlanta, USA. ${ }^{2}$ Centers for Disease Control and Prevention, Division of Reproductive Health (CDCF Contractor), Atlanta, USA. ${ }^{3}$ EngenderHealth, Washington, DC, USA. ${ }^{4}$ Regional Medical Officer, Kigoma, Kigoma Region, Tanzania. ${ }^{5}$ AMCA Inter Consult, Dar es Salaam, Tanzania.

\section{Received: 15 April 2018 Accepted: 17 August 2018} Published online: 12 September 2018

\section{References}

1. WHO. Trends in maternal mortality. 1990 To 2015. Estimates by WHO UNICEF, UNFPA, the World Bank \& the UN population division. Geneva: World Health Organization; 2015.

2. Chola L, McGee S, Tugendhaft A, Buchmann E, Hofman K. Scaling up family planning to reduce maternal and child mortality: the potential costs and benefits of modern contraceptive use in South Africa. PLoS One. 2015;10(6): e0130077. https://doi.org/10.1371/journal.pone.0130077.

3. Ahmed S, Li Q, Liu L, Tsui A. Maternal deaths averted by contraceptive use: an analysis of 172 countries. Lancet. 2012;380(9837):111-25.

4. Collumbien M, Gerressu M, Cleland J. Non-use and use of ineffective methods of contraception. In: Comparative quantification of health risks: global and regional burden of disease attributable to selected major risk factors. Geneva: WHO; 2004. p. 1255-320. ISBN 9241580313.
5. Ganatra B, Faundes A. Role of birth spacing, family planning services, safe abortion services and post-abortion care in reducing maternal mortality. Best Pract Res Clin Obstet Gynaecol. 2016;36:145-55. https://doi.org/10. 1016/j.bpobgyn.2016.07.008.

6. Rutstein SO. Effects of preceding birth intervals on neonatal, infant and under-five years mortality and nutritional status in developing countries: evidence from the demographic and health surveys. Int J Gynaecol Obstet. 2005;89(Suppl 1):S7-24.

7. Tumlinson K, Okigbo CC, Speizer IS. Provider barriers to family planning access in urban Kenya. Contraception. 2015;92(2):143-51. ISSN 0010-7824

8. Ministry of Health, Community Development, Gender, Elderly and Children (MoHCDGEC) [Tanzania Mainland], Ministry of Health (MoH) [Zanzibar], National Bureau of Statistics (NBS), Office of the Chief Government Statistician (OCGS), and ICF. 2016. Tanzania Demographic and Health Survey and Malaria Indicator Survey (TDHS-MIS) 2015-16. Dar es Salaam, Tanzania, and Rockville, Maryland, USA: MoHCDGEC, MoH, NBS, OCGS, and ICF.

9. Bruce J. Fundamental elements of quality of care: a simple framework. Stud Fam Plan. 1990;21(2):61-91.

10. Zapata LB, Tregear SJ, Curtis KM, Tiller M, Pazol K, Mautone-Smith N, Gavin LE. Impact of contraceptive counseling in clinical settings: A systematic review. Am J Prev Med. 2015;49(2 suppl 1):S31-45. https://doi.org/10.1016/j. amepre.2015.03.023.

11. Tessema GA, Streak Gomersall J, Mahmood MA, Laurence CO. Factors determining quality of care in family planning services in Africa: a systematic review of mixed evidence. PLoS One. 2016;11(11):e0165627. https://doi.org/10.1371/journal.pone.0165627.

12. Achyut $P$, Mishra A, Montana L, Sengupta $R$, Calhoun LM, Nanda $P$. Integration of family planning with maternal health services: an opportunity to increase postpartum modern contraceptive use in urban Uttar Pradesh, India. J Fam Plann Reprod Health Care. 2016;42(2):107-15. https://doi.org/10. 1136/jfprhc-2015-101271

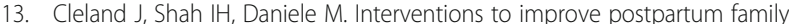
planning in low- and middle-income countries: program implications and research priorities. Stud Fam Plan. 2015:46(4):423-41.

14. Gaffield ME, Egan S, Temmerman M. It's about time: WHO and partners release programming strategies for postpartum family planning. Global Health, Science and Practice. 2014;2:4-9. https://doi.org/10.9745/GHSP-D-13-00156

15. Lopez LM, Grey TW, Hiller JE, Chen M. Education for contraceptive use by women after childbirth (review). Cochrane Database Syst Rev. 2015;7: CD001863. https://doi.org/10.1002/14651858.CD001863.pub4

16. Speizer IS, Fotso JC, Okigbo C, Faye CM, Seck C. Influence of integrated services on postpartum family planning use: a cross-sectional survey from urban Senegal. BMC Public Health. 2013;13:752.

17. Yadav D, Dhillon P. Assessing the impact of family planning advice on unmet need and contraceptive use among currently married women in Uttar Pradesh, India. PLoS One. 2015;10(3):e0118584. https://doi.org/10.1371/journal.pone.0118584.

18. Adanikin A, Onwudiegwu U, Loto O. Influence of multiple antenatal counselling sessions on modern contraceptive uptake in Nigeria. Eur $J$ Contracept Reprod Health Care. 2013;18(5):381-7.

19. Ayiasi RM, Muhumuza C, Bukenya J, Orach G. The effect of prenatal counselling on postpartum family planning use among early postpartum women in Masindi and Kiryandongo districts, Uganda. Pan Afr Med J. 2015;21(138)

20. Vural F, Vural B, Cakiroglu Y. The effect of combined antenatal and postnatal counselling on postpartum modern contraceptive use: prospective casecontrol study in Kocaeli, Turkey. J Clin Diagn Res. 2016:10(4):OC04-7.

21. Akman M, Tuzun S, Uzuner A, Basgul A, Kavak Z. The influence of prenatal counselling on postpartum contraceptive choice. J Int Med Res. 2010;38(4):1243-9.

22. Vance G, Janowitz B, Chen M, Boyer B, Kasonde P, Asare G, Kafulubiti B, Stanback $J$. Integrating family planning messages into immunization services: a clusterrandomized trial in Ghana and Zambia. Health Policy Plan. 2014;29:359-66.

23. Cooper CM, Fields R, Mazzeo Cl, Taylor N, Pfitzer A, Momolu M, JabbehHowe C. Successful proof of concept of family planning and immunization integration in Liberia. Glob Health: Sci Pract. 2015:3(2):71-84.

24. Centers for Disease Control and Prevention. Health facility assessment of emergency obstetric and neonatal Care Services in Kigoma Region, Tanzania: selected findings, 2016. Atlanta, GA: centers for disease control and Prevention; 2016.

25. Mody SK, Nair S, Dasgupta A, Raj A, Donta B, Saggurti N, Naik DD, Silverman JG. Postpartum contraception utilization among low-income women seeking immunization for infants in Mumabi, India. Contraception. 2014;89:516-20. 
26. Eluwa GIE, Atamewalen R, Odogwu K, Babatunde A. Success providing postpartum intrauterine devices in private-sector health care facilities in Nigeria: factors associated with uptake. Glob Health: Sci Pract. 2016;4(2):276-83.

27. Dulli LS, Eichleay M, Rademacher K, Sortijas S, Nsengiyumva T. Meeting postpartum women's family planning needs through integrated family planning and immunization services: results of a cluster-randomized controlled trial in Rwanda. Glob Health: Sci Pract. 2016;4(1):73-86.

28. Sedgh G, Hussain R. Reasons for contraceptive nonuse among women having unmet need for contraception in developing countries. Stud Fam Plan. 2014;45(2):151-69.

29. Calhoun LS, Speizer IS, Rimal R, Sripad P, Chatterjee N, Achyut P, Nanda P. Provider imposed restrictions to clients' access to family planning in urban Uttar Pradesh, India: a mixed methods study. BMC Health Serv Res. 2013;13:532.

30. Sidze EM, Lardoux S, Speizer IS, Faye CM, Mutua MM, Badji F. Young women's access to and use of contraceptives: the role of providers' restrictions in urban Senegal. Int Perspect Sex Reprod Health. 2014; 40(4):176-83.

31. Benson LS, Perrucci A, Drey EA, Steinauer JE. Contraception. 2012;85(2):198203. https://doi.org/10.1016/j.contraception.2011.05.021.

32. Tumlinson K, Pence BW, Curtis SL, Marshall SW, Speizer IS. Quality of care and contraceptive use in urban Kenya. Int Perspect Sex and ReproHealth. 2015;41(2):69-79. https://doi.org/10.1363/4106915.

33. Centers for Disease Control and Prevention 2016. Kigoma Reprod Health Survey: Kigoma Region, Tanzania. Atlanta, GA: Centers for Disease Control and Prevention; 2017.

34. National Bureau of Statistics \& Office of Chief Government Statistician. The United Republic of Tanzania, Kigoma region: basic demographic and socioeconomic profile. In: 2012 population and housing census; 2016.

35. Filmer D, Pritchett LH. Estimating wealth effects without expenditure data-or tears: an application to educational enrollments in states of India. Demography. 2001;38(1):115-32. PubMed PMID: 11227840

36. Rauh-Benoit LA, Tepper NK, Zapata LB, Whiteman MK, Curtis KM, Mandel MG, Marchbanks PA, Jamieson DJ. Healthcare provider attitudes of safety of intrauterine devices in the postpartum period. J Women's Health. 2017; 26(7):768-73. https://doi.org/10.1089/jwh.2016.5985.

37. Schwandt HM, Speizer IS, Corroon M. Contraceptive service provider imposed restrictions to contraceptive access in urban Nigeria. BMC Health Serv Res. 2017;17:268. https://doi.org/10.1186/s12913-017-2233-0.

38. Hipp S, Carlson A, McFarlane E. Improving reproductive life planning in Hawai'i: one key question. Hawaii J Med Public Health. 2017;76(9):261-4.

Ready to submit your research? Choose BMC and benefit from:

- fast, convenient online submission

- thorough peer review by experienced researchers in your field

- rapid publication on acceptance

- support for research data, including large and complex data types

- gold Open Access which fosters wider collaboration and increased citations

- maximum visibility for your research: over $100 \mathrm{M}$ website views per year

At BMC, research is always in progress.

Learn more biomedcentral.com/submissions 